\title{
Differences in the Microarchitectural and Histomorphologic Characteristics Between Glucocorticoid-induced Osteonecrosis of Femoral Head and Alcohol-induced Osteonecrosis of Femoral Head
}

\section{Yiwei Chen}

Shanghai Sixth People's Hospital: Shanghai 6th Peoples Hospital Affiliated to Shanghai Jiaotong University

\section{Kexin Liu}

Shanghai Sixth People's Hospital: Shanghai 6th Peoples Hospital Affiliated to Shanghai Jiaotong University

\section{Yu Miao}

Shanghai Sixth People's Hospital: Shanghai 6th Peoples Hospital Affiliated to Shanghai Jiaotong University

\section{Bin Zhu}

Nanjing Medical University affiliated Nanjing Hospital: Nanjing First Hospital

\section{Feng Xue}

Shanghai Sixth People's Hospital: Shanghai 6th Peoples Hospital Affiliated to Shanghai Jiaotong University

\section{Junhui Yin}

Shanghai Sixth People's Hospital: Shanghai 6th Peoples Hospital Affiliated to Shanghai Jiaotong University

\section{Minghao Zheng}

The University of Western Australia

guangyi li ( $\sim$ guangyi.li@shsmu.edu.cn )

Shanghai Sixth Peoples Hospital

\section{Changqing Zhang}

Shanghai Sixth People's Hospital: Shanghai 6th Peoples Hospital Affiliated to Shanghai Jiaotong University

\section{Research article}


Keywords: Osteonecrosis of the femoral head, glucocorticoid, alcohol, micro-computed tomography, microstructure, bone histology, bone remodeling

Posted Date: September 29th, 2021

DOI: https://doi.org/10.21203/rs.3.rs-934179/v1

License: (c) (1) This work is licensed under a Creative Commons Attribution 4.0 International License. Read Full License 


\section{Abstract}

Aims

To analyze microarchitecture and histomorphology characteristics of different regions in femoral heads from patients with glucocorticoid-induced osteonecrosis of femoral head (GIONFH) and alcohol-induced osteonecrosis of femoral head (AIONFH).

Methods

Patients diagnosed with GIONFH and AIONFH were recruited. Femoral heads were obtained after total hip replacement. Micro-CT was applied to evaluate the microstructure of 9 regions of interest (ROIs) in the femoral head. Along the supero-inferior orientation, the femoral head was divided into necrotic region, reactive interface, and normal region; along the medio-lateral orientation, the femoral head was divided into medial region, central region and lateral region. Decalcified and undecalcified bone histology were then performed to assess histopathological alterations and bone remodeling levels.

Results

42 GIONFH patients (50 hips) and 43 AIONFH patients (50 hips) anticipated in the study. In the necrotic region, most of the microarchitectural parameters did not differ significantly between GIONFH and AIONFH, whereas both the reactive interface and normal region illustrated significant differences in the microstructure and histomorphometry. The reactive interface and normal region exhibited a less sclerotic microarchitecture, but a higher bone remodeling level in GIONFH as compared with AIONFH. Despite similar necrotic pathological manifestations, subchondral trabecular microfracture in the necrotic region was more severe and vasculature of the reactive interface was more abundant in GIONFH.

Conclusions

Although these two subtypes of ONFH shared similar microarchitecture and pathological features in the necrotic region, GIONFH exhibited a less sclerotic microarchitecture and a more active bone metabolic status in both the reactive interface and normal region.

\section{Introduction}

Osteonecrosis of femoral head (ONFH) is known as avascular necrosis or aseptic necrosis of the femoral head, associated with an interruption of the blood supply ${ }^{1}$. One of the pathological features of ONFH is the damage of subchondral vascular microcirculation, which leads to osteonecrosis, eventually resulting in repeated microfractures in the femoral head and articular collapse ${ }^{2}$. In the United States, about 10,000 to 20,000 patients are newly diagnosed with the disease annually ${ }^{3}$. In a recent nationally representative survey, the cumulative number of patients with non-traumatic ONFH was estimated to be around 8.12 million among the Chinese population ${ }^{4}$. As a devastating disease frequently leading to secondary hip 
arthritis, ONFH imposes a heavy economic burden on the whole society ${ }^{5,6}$. Most patients end up having to choose total hip replacement (THA) ${ }^{7}$, and younger patients undergoing THA may require further revision of hip arthroplasty, which will lead to increased morbidity ${ }^{8}$.

The etiology and pathogenesis of nontraumatic ONFH are not well understood ${ }^{9}$. Long-term use of glucocorticoid, alcohol abuse, genetic mutations, and thrombotic/fibrinolytic diseases have been identified as possible risk factors for ONFH ${ }^{10,11}$. Glucocorticoid usage and alcohol abuse are the most widely reported risk factors for nontraumatic $\mathrm{ONFH}{ }^{12}$. Glucocorticoid is the most common cause of nontraumatic osteonecrosis $(51 \%)^{11}$, and this was followed by alcohol, which is more than one in five ${ }^{13}$. Glucocorticoid may have a complex relationship with lipid metabolism and vascular endothelium in the pathogenesis of glucocorticoid-induced osteonecrosis of femoral head (GIONFH) ${ }^{14}$, possibly affecting the number and function of circulating endothelial progenitor cells, which are necessary for vascular repair and new angiogenesis ${ }^{15}$. In addition, glucocorticoid has the potential effect on directing precursor cells towards an adipogenic or osteogenic pathway ${ }^{16}$. There is a hypothesis that abnormal alcohol metabolism may lead to the deterioration of bone tissue in the femoral head through toxic by-products such as acetaldehyde, free radicals and acetaldehyde adducts ${ }^{17}$, with the potential mechanism of intravascular coagulation and coagulation cascade regulation ${ }^{18}$. Alcohol could also disrupt osteogenic differentiation and promotes lipogenesis of bone marrow mesenchymal stem cells, in the pathogenesis of alcohol-induced osteonecrosis of femoral head (AIONFH) ${ }^{19,20}$.

Using histological analysis, Kim et al. found that patients with idiopathic ONFH or AIONFH have normal or nearly normal cancellous bone in the acetabulum and proximal femur, whereas GIONFH might be associated with more extensive osteonecrosis ${ }^{21}$. Patients with ONFH secondary to sickle-cell disease or steroid use have been found to have poor results of total hip arthroplasty when compared with agematched controls ${ }^{22-26}$. This heterogeneity may be related to the different biological effects on the bone tissue induced by different risk factors. However, in most histopathological studies on ONFH, only regular decalcified sections and hematoxylin-eosin (H\&E) staining were applied, in which mineralization and bone remodeling status cannot be assessed using histology or imaging mass spectrometry. Undecalcified bone histology shows tremendous importance in a variety of bone research areas, including metabolic bone diseases and disorders of mineral metabolism ${ }^{27}$. Undecalcified sections and related trichrome stains could demonstrate both the mineralized and cellular components of bone tissue, and provide pivotal information on bone turnover ${ }^{28}$, which have not been widely applied in the histopathological analysis of ONFH ${ }^{29,30}$.

The morphology of the femoral head is unique, and the structure of bone trabecula is complicated and heterogeneous ${ }^{31}$. Bone microstructure plays an important role in the pathogenesis and development of $\mathrm{ONFH}^{32,33}$. Micro-computed tomography (micro-CT) has been introduced to perform a three-dimensional evaluation of the detailed microstructure in the femoral head with osteonecrosis. However, most of these previous studies only focused on selective specimens with small diameters, which were extracted from 
specific regions of the proximal femur ${ }^{34}$. The overall bone microstructure of the whole femoral head with osteonecrosis, which is of great clinical significance, is not frequently evaluated in early studies. The microarchitectural analysis will deepen our understanding of the detailed overall morphological alterations of the whole femoral head in the development of ONFH, and help clinicians reevaluate the severity level of the disease, which might modify the indication for jointpreservation surgery for ONFH.

Despite distinct etiologies between GIONFH and AIONFH, the differences of the microarchitectural and histomorphologic characteristics between these subtypes of ONFH are rarely investigated. Accordingly, we hypothesized that the microstructural and histomorphometric characteristics of the femoral head in GIONFH and AIONFH are different. To address this, we assessed microarchitecture, bone remodeling activity and histopathological alterations of trabecular bone in different regions of the femoral head from patients with GIONFH and AIONFH, using micro-CT, decalcified and undecalcified bone histology.

\section{Methods}

Subjects

Between September 2015 and October 2019, 85 patients diagnosed with ONFH who underwent THA surgery, were recruited in the study. These patients who participated in this study were divided into two groups based upon which etiology they belonged to: (1) glucocorticoid-induced osteonecrosis of femoral head (GIONFH) or (2) alcohol-induced osteonecrosis of femoral head (AIONFH). Patients were recruited to the GIONFH group if they met the classification criteria of glucocorticoid-induced osteonecrosis of femoral head included the following: (1) Patients should have a history of glucocorticoid use more than 2 $\mathrm{g}$ of prednisolone or its equivalent within 3 months;(2) ONFH was diagnosed within 2 years after glucocorticoid administration;(3) There were no other risk factors except glucocorticoids ${ }^{35,36}$. Regarding AIONFH, we collected information about age at starting and cessation of alcohol consumption, usual frequency of drinking, and the usual volume of alcohol intake by beverage type. This volume was consequently converted to grams of ethanol and values for each beverage type were added. The ethanol content for calculation was as follows: $4.5 \%$ for beer, $12 \%$ for wine, $43 \%$ for whisky, $15.5 \%$ for rice wine and $50 \%$ for baijiu (a distilled alcoholic beverage made in China) ${ }^{37}$. We described habitual drinking in patients with alcohol-associated ONFH as more than 3032 drink years of the cumulative alcohol intake according to the previous report ${ }^{37}$. The cumulative drinking amount termed as 'drink-years' was calculated by multiplying the weekly ethanol consumption (gram) by the total number of years of drinking. Patients recruited to the AIONFH group should also meet classification criteria of alcoholassociated ONFH: (1) patients should have a history of alcohol intake of more than $400 \mathrm{ml} /$ week of pure ethanol (320 g/week of any type of alcoholic beverage) for more than 6 months;(2) ONFH was diagnosed within 1 year after this dose of alcohol intake;(3) There should be no other risk factors ${ }^{38}$.

Exclusion criteria for ONFH patients were as follows: (1) other known metabolic or bone disorders which could affect bone metabolism, such as thyroid or parathyroid disease, and malignancy; (2) receiving 
other treatments that affect bone metabolism such as anti-resorptive drugs, calcitonin, thyroid or parathyroid hormone therapy, or hormonal replacement therapy; or (3) history of hip fracture and osteotomy. Information about glucocorticoid usage, alcohol consumption, smoking in two groups is shown in Table 1. All cases were classified with different clinical stages recommended by the Association Research Circulation Osseous (ARCO) ${ }^{39}$. The cases in this study were all ARCO stage II, IIIA, IIIB or IV, with severe pain or severe hip dysfunction (Fig. 1). Given that location of the necrosis may affect topographical observations, the Japanese classification is important to understand changes, particularly addressing lateral extension. Therefore, we use the Japanese classification to classify all cases as well. Type A lesions account for only a third or less of the weight-bearing portion. Type B lesions accounted for two-thirds or less of the weight-bearing portion. Both type $\mathrm{C} 1$ and $\mathrm{C} 2$ lesions account for more than two thirds of the medial weight bearing portion of the acetabulum, but $\mathrm{C} 2$ lesions extend laterally to the acetabulum margin, while $\mathrm{C} 1$ lesions do not ${ }^{40}$. Informed consent was obtained from each patient. The study protocol was approved by the Human Research Ethics committee of our hospital and complied with the Declaration of Helsinki.

\section{Micro-CT examination}

Femoral head specimens were obtained after THA surgery and stored in a $-80^{\circ} \mathrm{C}$ freezer. Then, they were scanned using the high resolution micro-CT system ( $\mu$ CT100, Scanco Medical, Bassersdorf, Switzerland), with the following scan settings: $X$-ray tube voltage, $70 \mathrm{kVp}$; tube current, $200 \mu \mathrm{As}$; exposure time, $300 \mathrm{~ms}$ ; projection number, 1000; and voxel size, $73.6 \mu \mathrm{m}^{41}$. The scan length was approximately $49 \mathrm{~mm}$, resulting in 1200 slices and a 45-minute scan time. Orientation adjustment of the femoral head, segmentation of the regions of interest (ROIs), and measurement of bone microstructural parameters in each region were performed by the built-in software. Orientations of all femoral heads were adjusted based on anatomical landmarks using the fovea capitis femoris and the main trabeculae direction (MTD), where the principal compressive trabeculae were located (Fig. 2A) ${ }^{31,42} .9$ ROIs were set in the central coronal plane of the femoral head (Fig. 2B). Specifically, along the supero-inferior orientation, the femoral head was divided into three main regions with reference to the boundary of the necrotic lesion: superior region (necrotic region), central region (i.e. reactive interface or necrotic-normal junction region), and inferior region (normal region); along the medio-lateral orientation, the femoral head was divided into another three main regions with reference to MTD: medial region, central region (principal compressive trabeculae region) and lateral region. Thus, a total of nine ROls (Sup-Med, SupCen, Sup-Lat, Cen-Med, Cen-Cen, Cen-Lat, Inf-Med, Inf-Cen, and Inf-Lat) were included in the microstructure analysis. In each ROI, a cylindrical bone specimen ( $5 \mathrm{~mm}$ in height and $5 \mathrm{~mm}$ in diameter) was set for analysis (Fig. 2C).

After scanning and reconstruction, the images were transferred with a fixed threshold to binary images; color images were also produced based on X-ray attenuation coefficient values, representing mineralization distribution in the trabeculae (Fig. 3-4). Bone microarchitecture parameters were calculated. In specimens with cystic lesions and patchy necrotic debris, the measurement for bone 
microarchitecture was only conducted in the trabecular region in which the cysts and debris were excluded.

The following microarchitectural parameters were measured: bone volume fraction (BV/TV) (\%), bone surface/volume ratio (BS/BV) $(1 / \mathrm{mm})$, trabecular thickness (Tb.Th) $(\mu \mathrm{m})$, trabecular separation (Tb.Sp) $(\mu \mathrm{m})$, trabecular number (Tb.N) $(1 / \mathrm{mm})$, structure model index (SMI), degree of anisotropy (DA), connectivity density (Conn.D) $(1 / \mathrm{mm} 3)^{43}$.

Specimen preparation

After micro-CT scanning, all femoral heads were cut in half with a diamond saw, coronally separating the anterior hemisphere from the posterior hemisphere (Fig. 3A-C). A 10-mm-thick coronal bone slice was then sectioned from each femoral head hemisphere. One slice was prepared for decalcified bone section, and the other slice was used for undecalcified bone histology. Each slice was divided into nine ROls as aforementioned. All these specimens were fixed in paraformaldehyde for 2 weeks, which was followed by the related process for undecalcified or decalcified histology. Decalcified samples were embedded in paraffin wax, sectioned into 5 - $\mu$ m-thick slices and stained with H\&E. Undecalcified samples were embedded in methyl-methacrylate, sectioned into 5 - $\mu \mathrm{m}$-thick slices, and were stained by Goldner's Trichrome method.

Histopathological assessment and histomorphometry

The decalcified sections were examined for the evidence of osteonecrosis and secondary pathologic changes. For the undecalcified sections, histomorphometry was performed using Bioquant Osteo Histomorphometry software (Bioquant Osteo, Nashville, TN, USA). The following bone formation and resorption parameters were measured in each ROI: thickness of osteoid $(0 . T h, \mu \mathrm{m})$, percentage osteoid volume (OV/BV) (\%), percentage osteoid surface (OS/BS) (\%), specific osteoid surface (OS/BV) $\left(\mathrm{mm}^{2} / \mathrm{mm}^{3}\right)$, percentage eroded surface (ES/BS) (\%), specific eroded surface $(\mathrm{ES} / \mathrm{BV})\left(\mathrm{mm}^{2} / \mathrm{mm}^{3}\right)$ and eroded surface in bone tissue volume (ES/TV) $\left(\mathrm{mm}^{2} / \mathrm{mm}^{3}\right)^{44}$.

Statistical analysis

The Kolmogorov-Smirnov test was used to determine if parameters followed a normal (Gaussianshaped) distribution and no significant departures were identified. The unpaired Student's t test was used to compare all microarchitecture and bone remodeling parameters between groups. A conservative twotailed $\mathrm{P}<0.05$ was chosen a priori to declare a statistically significant result to account for multiple comparisons and to avoid type I errors ${ }^{45}$. Analysis of data was performed using the Statistics Package for Social Sciences (SPSS for Windows, version 17.0; SPSS Inc, Chicago, IL, USA). Continuous data are presented as mean \pm standard deviation (SD). Gender, smoker, diabetes, hypertension, side of ONFH, ARCO stage $^{46}$, Japanese Classification System ${ }^{40}$ were compared between GIONFH and AIONFH groups using Fisher's exact test, Chi square or Chi square test with Yates correction, as appropriate. 
Table 1 Characteristics of the study participants

Data are the median (interquartile range) for quantitative variables and no. (\%) of patients for qualitative variables. Bold indicates statistically significant difference.

\section{Results}

Subject characteristics

A total of 85 patients (100 hips) diagnosed with ONFH were recruited in the study. 42 GIONFH patients (50 hips) had various indications for glucocorticoid treatment, including autoimmune diseases [systemic lupus erythematosus $(n=15)$, rheumatoid arthritis $(n=2)$ ], renal diseases [nephritic syndrome $(n=5)$, pyelonephritis $(n=1)$, IgA nephropathy $(n=2)$, chronic nephritis $(n=2)$ and renal transplantation $(n=1)]$, dermatogic diseases [psoriasis $(n=1))$, skin pruritus $(n=1)$, eczema $(n=2)$, urticaria $(n=1)$, and neurodermatitis $(n=2)]$, thrombocytopenic purpura $(n=2)$, chronic pulmonary fibrosis $(n=1)$, craniopharyngioma $(n=1)$, myocarditis $(n=1)$, bronchial asthma $(n=2) .43$ participants (50 hips) with alcohol-associated ONFH were classified into the AIONFH group. Patients who were diagnosed with bilateral ONFH are all at different stages.

GIONFH and AIONFH subjects were well matched on demographic factors and medical conditions (Table 1), with the exception being the proportion of males, which was significantly higher in the AIONFH group than that in the GIONFH group $(P<0.0001)$.

Comparative analysis of microarchitecture in different regions between GIONFH and AIONFH

In Sup-Med region, none of the microarchitecture parameters differed significantly between GIONFH and AIONFH, with the exception of DA. There were significantly lower values of DA in GIONFH, compared to AIONFH. Similarly, in Sup-Cen and Sup-Lat regions, none of the microarchitecture parameters differed significantly between GIONFH and AIONFH, with the exception of SMI (Fig. 4-5). There were significantly higher values of SMI in GIONFH, compared to AIONFH.

In Cen-Med region (Fig. 4-5), BS/BV, Tb.Th, Conn.D and DA were not significantly different between GIONFH and AIONFH. However, there were significantly lower values of BV/TV and Tb.N, higher values of Tb.Sp and SMI in GIONFH, compared to AIONFH.

In Cen-Cen region, all the microarchitecture parameters were significantly different between GIONFH and AIONFH, with the exception of DA (Fig. 4-5). Compared to AIONFH, there were significantly higher values of BS/BV, Tb.Sp, Conn.D and SMI in GIONFH, with significantly lower values of BV/TV, Tb.Th, Tb.N.

In Cen-Lat region, Tb.Th, Conn.D, SMI and DA were not significantly different between GIONFH and AIONFH (Fig. 4-5). Nevertheless, there were significantly lower values of BV/TV and Tb.N, higher values of BS/BV and Tb.Sp in GIONFH than in AIONFH. 
Characteristics of study participants

Number of patients

Gender (male/female)

Age (years)

BMI $\left(\mathrm{kg} / \mathrm{m}^{2}\right)$

Smoker n (\%)

Diabetes

Hypertension

\section{Characteristics of osteonecrosis}

Number of hips

Side (right: left)

Timing since the onset of the ONFH, months

\section{Glucocorticoid dosage}

Glucocorticoid pulse therapy, n (\%)

Duration of glucocorticoid administration, years

\section{Glucocorticoid treatment}

Drug species
42

$22 / 20$

47.29(34.75-45)

24.17(22.42-26.51)

19囚45.23》

4

8
43

$42 / 1$

$<0.0001$

0.1610

0.5187

24.83(22.65-

27.56)

26『60.47》

0.1597

4

0.9721

9

0.8283

$\begin{array}{ll}4 & 0.9721 \\ 9 & 0.8283\end{array}$

50

$24: 26$

27.05(8-18)

14(33)

9.12(7-10)

NA

NA

20:30

24.12(8-12)

0.5459

0.8168

50 


\section{Type A}

Type B

Type C1

Type C2

1

6

6
2
22

21

In Inf-Med region, there were lower values of BV/TV, higher values of BS/BV and SMI in GIONFH than in AIONFH, while the other parameters showed no significant difference. In Inf-Cen region, BS/BV, Tb.Th, Conn.D and DA were not significantly different between GIONFH and AIONFH. However, there were significantly lower values of BV/TV and Tb.N, higher values of Tb.Sp and SMI in GIONFH, compared to AIONFH. In Inf-Lat region, none of the microarchitecture parameters differed significantly between GIONFH and AIONFH, except for Tb.N (Fig. 4-5).

Histological assessment of osteonecrosis in both groups

In the necrotic region of the femoral head, bone marrow necrosis and osteocytic death were observed in both groups. Hematopoietic cells disappeared and adipose nucleus staining was absent in the bone marrow, indicating the necrosis of bone marrow (Fig. 7). The necrotic lesions were not scattered and patchy in the bone marrow space, but exhibiting as a large continuous area with different shapes. In addition, most bone trabeculae represented empty osteocyte lacunae, seldom encompassing some pycnotic nuclei of osteocytes. In the subchondral trabecular bone of the necrotic region, microfracture and patchy debris was frequently observed, which was more obvious and severe in GIONFH.

In the necrotic-normal junction region, reparative process, such as vascular-rich granulation tissue, was observed. Histiocytes and giant cells aggregated in the reactive fibrous interface, especially in the lateral aspect. Although vessels penetrated into the fibrotic capsule, the angiogenesis was blocked at the boundary of the sequestrum and reparative fibrovascular tissue could not penetrate into the bone marrow space of the necrotic region. Constantly, the reparative fibrovascular tissue would convert to a sclerotic rim in the late stages of the disease, which was attributed to the bone formation by osteoblasts.

Compared to AIONFH, the angiogenesis was more abundant in the reactive interface in GIONFH, bringing about a more active bone remodeling status, despite the failure of thorough reparative substitution of the sequestrum in both groups (Fig. 7).

Comparative analysis of bone remodeling levels between GIONFH and AIONFH

Due to the devitalization status of the necrotic region, the Sup-Med, Sup-Cen and Sup-Lat regions were not analyzed in the aspect of bone remodeling levels. 
In the necrotic-normal junction region, including Cen-Med, Cen-Cen and Cen-Lat regions, all the bone remodeling parameters were significantly higher in GIONFH, compared with AIONFH (Fig. 6, Fig. 8). There were higher values of O.Th, OV/BV, OS/BS and OS/BV, indicating a more active bone formation status. Bone resorption activity was also higher, as suggested by higher values of erosion parameters including ES/BS, ES/BV and ES/TV.

In Inf-Med region, most bone remodeling parameters were significantly higher in GIONFH than AIONFH, with the exception of ES/BV (Fig. 6, Fig. 8). In Inf-Cen region, the bone formation parameters 0.Th, OV/BV, OS/BS and OS/BV were significantly elevated in GIONFH samples than those in AIONFH (Fig. 6, Fig. 8). The bone resorption parameter ES/BS was significantly elevated in GIONFH. No significant differences were observed in ES/BV and ES/TV. In Inf-Lat region, there were higher values of 0.Th, OV/BV, OS/BS in GIONFH, indicating a more active bone formation status (Fig. 6, Fig. 8). OS/BV were also higher in GIONFH, although no significant difference was observed. The bone resorption parameter ES/BS was significantly elevated in GIONFH. No significant differences were observed in ES/BV and ES/TV.

\section{Discussion}

In this study, we analyzed simultaneously the microarchitecture, histopathologic alterations and bone remodeling levels in different regions of the involved femoral head between GIONFH and AIONFH. The two groups shared similar microarchitectural characteristics in the necrotic region, whereas both the reactive interface and normal regions illustrated significant differences in the microstructure and histomorphometry. The reactive interface and normal regions exhibited a less sclerotic microarchitecture, but a higher bone remodeling level in GIONFH as compared with AIONFH. Despite similar necrotic pathological manifestations, subchondral trabecular microfracture and debris was more severe in the necrotic region, and the reactive interface was more vascular in GIONFH.

Microstructure is an important component of bone quality, and its integrity contributes to the maintenance of bone strength ${ }^{47,48}$. The microarchitectural patterns in different regions of the joint may differ, reflecting different types and levels of biomechanical loads ${ }^{49,50}$. Most of the stress on the femoral head is borne by the principal compressive trabeculae in daily activities, exhibiting compact microarchitecture and high biomechanical strength ${ }^{51}$. Our results revealed that the reactive interface and normal region displayed a less sclerotic microarchitecture in GIONFH as compared with AIONFH, exhibiting a lower bone volume fraction, less trabecular number, and wider trabecular space, especially in the Cen-Cen region. The deteriorated microarchitecture has been reported to play a pivotal role in the failure of mechanical stabilization in the femoral head, and eventually leads to the progression of collapse ${ }^{52}$. The alterations of microstructure may be due to the distinct bone remodeling status ${ }^{53}$, which could be influenced by different reagents that affect bone metabolism such as glucocorticoid and alcohol.

Our study demonstrated that the reactive interface and normal region exhibited higher bone remodeling levels in GIONFH, despite a less sclerotic microarchitecture observed. This phenomenon might be due to 
the possibility that the activity of osteoclast-mediated bone resorption in GIONFH was much higher, overweighing the effect of the elevated level of bone formation activity. This variation might be attributed to the different biological responses of relevant bone cells trying to repair necrotic tissue ${ }^{54}$, reflecting distinct effects of these two etiologies. It has been proposed that glucocorticoid and alcohol reduce femoral artery perfusion through mechanisms such as vascular endothelial injury and microvascular thrombosis ${ }^{55,56}$. They can also induce the formation of intramedullary fat, which increases the pressure in the bones, leading to venous congestion and blocked arteries ${ }^{19,57,58}$. Glucocorticoid has been reported to reduce the production of osteoblasts, increase the apoptosis of osteoblasts and prolong the life of osteoclasts ${ }^{16,59}$. Accumulating evidence has also shown that heavy alcohol consumption impairs bone homoeostasis, which disturbs the osteoblast formation and proliferation, and promotes osteoclastogenesis ${ }^{60,61}$. Despite the adverse effect on bone metabolism by both glucocorticoid and alcohol, glucocorticoid might illustrate a more negative interference on osteogenesis and reparative process in the condition of ONFH. Chernetsky et al. found that histologic alterations of necrosis and repair evolved in a given sequence after bone death, the rate of which was faster in the GIONFH ${ }^{62}$. In a study by Wei et al. ${ }^{63}$, GIONFH was characterized by multiple "osteolytic bone destruction", while AIONFH was manifested by some kinds of "coagulative destruction".

Both GIONFH and AIONFH showed a large continuous area of bone marrow necrosis, osteocytic death and disordered subchondral trabecular structure in the necrotic region, with a fibrous reactive interface at the boundary of the sequestrum. The similar histologic features of necrosis between these two subtypes of ONFH was consistent the previous study by Chernetsky et al. ${ }^{62}$. The difference between the two groups was that the microstructure and arrangement of subchondral trabeculae in the necrotic region of GIONFH were more seriously damaged, with a more vascularized fibrous interface. The more deteriorated and chaotic subchondral microarchitecture might be attributed to the biomechanically weak strength of trabeculae and excessive osteoclast activity, induced by prolonged overdose usage of glucocorticoid in the condition of weight-bearing. Angiogenesis is an important part of the reparative response following necrosis ${ }^{64}$. Despite detrimental effects on bone angiogenesis by glucocorticoid in the pathogenesis of osteonecrosis, introduction of glucocorticoid could also promote angiogenesis under certain conditions ${ }^{65}$, which might be the explanation of the highly vascularized fibrous interface in GIONFH. The underlying mechanism needs further investigation in the future. High concentration of alcohol has been reported to show an adverse effect on neovascularization ${ }^{66,67}$, explaining the relatively less evident vascularization in the reactive interface in AIONFH.

There are several limitations in this study. One limitation of the present study was the absence of normal subjects. All of these bone specimens were obtained from patients with ONFH, without healthy controls. The relatively young age of these patients undergoing THA makes the acquisition and age matching of control samples difficult. But for all that, we cannot reveal whether there was a specific degree of heterogeneity in the microstructure of the femoral head in ONFH patients, even with the same trend in healthy controls. Second, this was a cross-sectional study using specimens of the femoral head, lacking 
dynamic bone remodeling parameters. Micro-CT scanning of the hip joint cannot be performed in vivo. Future advances in CT and MRI with improved spatial resolution may help solve this problem ${ }^{68-71}$.

In conclusion, GIONFH and AIONFH showed similar microarchitecture characteristics in the necrotic region, while the reactive interface and normal region exhibited a less sclerotic microarchitecture and a more active remodeling status in GIONFH than that in AIONFH. In the aspect of histopathology, microfracture of subchondral trabeculae in the necrotic region was more severe and vasculature of the reactive interface was more abundant in GIONFH. These phenomena might be due to the distinct biological effects of glucocorticoid and alcohol on bone metabolism and angiogenesis. This study provides a better understanding of microstructural, pathological and histomorphologic alterations in the whole femoral head with osteonecrosis induced by different risk factors, which might further contribute to the development of disease-modifying prevention strategies and therapeutics for ONFH, taking into etiologies.

\section{Abbreviations}

ONFH: Osteonecrosis of the femoral head; GIONFH: Glucocorticoid-induced osteonecrosis of femoral head; AIONFH: Alcohol-induced osteonecrosis of femoral head; ARCO: Association research circulation osseous; ROI: Regions of interest; THA: total hip replacement; micro-CT: Micro-computed tomography; H\&E: Hematoxylin-eosin; MTD: Main trabeculae direction; BV/TV: Bone volume fraction; BS/BV: Bone surface/volume ratio; Tb.Th: Trabecular thickness; Tb.Sp: Trabecular separation; Tb.N: Trabecular number; SMI: Structure model index; DA: Degree of anisotropy; Conn.D: Connectivity density; O.Th: Thickness of osteoid; OV/BV: Percentage osteoid volume; OS/BS: Percentage osteoid surface; OS/BV: Specific osteoid surface; OS/BS: Percentage eroded surface; ES/BV: Specific eroded surface; ES/TV: Eroded surface in bone tissue volume; SD: Standard deviation.

\section{Declarations}

\section{Acknowledgements}

Not applicable.

\section{Authors' contributions}

All authors listed have read and approved all versions of the manuscript YWC and GYL had full access to all of the data in the study and takes responsibility for the integrity of the data and the accuracy. Critical revision of the article for important intellectual content: YWC, KXL, YM, BZ, FX, JHY, MHZ, GYL, CQZ. All authors approved the final version to be submitted for publication.

\section{Authors' information}

Not applicable. 


\section{Funding}

This work was supported by the National Natural Science Foundation of China (grant no. 81702181) and the Projects of International Cooperation and Exchanges of National Natural Science Funding of China (grant no. 81820108020).

\section{Availability of data and materials}

The authors are committed to sharing their data, publishing the data, and making available the resources described in this publication to the scientific community.

\section{Ethics approval and consent to participate}

The study was conducted in accordance with the Declaration of Helsinki and International Conference on Harmonization Good Clinical Practice. The protocol and patient's informed consent received institutional review board/independent ethics committee approval prior to initiation of the study.

\section{Consent for publication}

Patient consent for publication was not required.

\section{Competing interests}

The authors declare that they have no competing interests.

\section{Author details}

1. Department of Orthopaedics, Shanghai Jiao Tong University Affiliated Sixth People's Hospital, Shanghai200233, China.

2. Institute of Microsurgery on Extremities, Shanghai Jiao Tong University Affiliated Sixth People's Hospital, Shanghai200233, China.

3. Department of Orthopedics, The First Affiliated Hospital of Nanjing Medical University, Nanjing210029, China.

4. Centre for Orthopaedic Research, School of Surgery, The University of Western Australia, Nedlands, WA 6009 , Australia.

\section{References}

1 Cui, Q. et al. ARCO Consensus on the Pathogenesis of Non-traumatic Osteonecrosis of the Femoral Head. J Korean Med Sci 36, e65, doi:10.3346/jkms.2021.36.e65 (2021). 
2 Aldridge, J. M., 3rd \& Urbaniak, J. R. Avascular necrosis of the femoral head: etiology, pathophysiology, classification, and current treatment guidelines. Am J Orthop (Belle Mead NJ) 33, 327332 (2004).

3 Mont, M. A., Cherian, J. J., Sierra, R. J., Jones, L. C. \& Lieberman, J. R. Nontraumatic Osteonecrosis of the Femoral Head: Where Do We Stand Today? A Ten-Year Update. J Bone Joint Surg Am 97, 1604-1627, doi:10.2106/JBJS.0.00071 (2015).

4 Zhao, D. W. et al. Prevalence of Nontraumatic Osteonecrosis of the Femoral Head and its Associated Risk Factors in the Chinese Population: Results from a Nationally Representative Survey. Chin Med J (Engl) 128, 2843-2850, doi:10.4103/0366-6999.168017 (2015).

5 Chen, C. H. et al. Alendronate in the prevention of collapse of the femoral head in nontraumatic osteonecrosis: a two-year multicenter, prospective, randomized, double-blind, placebo-controlled study. Arthritis Rheum 64, 1572-1578, doi:10.1002/art.33498 (2012).

6 Mont, M. A., Jones, L. C. \& Hungerford, D. S. Nontraumatic osteonecrosis of the femoral head: ten years later. J Bone Joint Surg Am 88, 1117-1132, doi:10.2106/jbjs.E.01041 (2006).

7 Olsen, M. et al. Total hip arthroplasty following failure of core decompression and tantalum rod implantation. Bone Joint J 98-b, 1175-1179, doi:10.1302/0301-620x.98b9.37252 (2016).

8 Steinberg, M. E. et al. Core decompression with bone grafting for osteonecrosis of the femoral head. Clin Orthop Relat Res, 71-78, doi:10.1097/00003086-200105000-00009 (2001).

9 Lavernia, C. J., Sierra, R. J. \& Grieco, F. R. Osteonecrosis of the femoral head. J Am Acad Orthop Surg 7, 250-261, doi:10.5435/00124635-199907000-00005 (1999).

10 Cohen-Rosenblum, A. \& Cui, Q. Osteonecrosis of the Femoral Head. Orthop Clin North Am 50, 139149, doi:10.1016/j.ocl.2018.10.001 (2019).

11 Shah, K. N., Racine, J., Jones, L. C. \& Aaron, R. K. Pathophysiology and risk factors for osteonecrosis. Curr Rev Musculoskelet Med 8, 201-209, doi:10.1007/s12178-015-9277-8 (2015).

12 Simank, H. G., Brocai, D. R., Brill, C. \& Lukoschek, M. Comparison of results of core decompression and intertrochanteric osteotomy for nontraumatic osteonecrosis of the femoral head using Cox regression and survivorship analysis. J Arthroplasty 16, 790-794, doi:10.1054/arth.2001.23580 (2001).

13 Chang, C., Greenspan, A. \& Gershwin, M. E. The pathogenesis, diagnosis and clinical manifestations of steroid-induced osteonecrosis. J Autoimmun 110, 102460, doi:10.1016/j.jaut.2020.102460 (2020).

14 Kerachian, M. A., Séguin, C. \& Harvey, E. J. Glucocorticoids in osteonecrosis of the femoral head: a new understanding of the mechanisms of action. J Steroid Biochem Mol Biol 114, 121-128, doi:10.1016/j.jsbmb.2009.02.007 (2009). 
15 Feng, Y. et al. Decreased in the number and function of circulation endothelial progenitor cells in patients with avascular necrosis of the femoral head. Bone 46, 32-40, doi:10.1016/j.bone.2009.09.001 (2010).

16 Tan, G., Kang, P. D. \& Pei, F. X. Glucocorticoids affect the metabolism of bone marrow stromal cells and lead to osteonecrosis of the femoral head: a review. Chin Med J (Engl) 125, 134-139 (2012).

17 Seamon, J., Keller, T., Saleh, J. \& Cui, Q. The pathogenesis of nontraumatic osteonecrosis. Arthritis 2012, 601763, doi:10.1155/2012/601763 (2012).

18 Pouya, F. \& Kerachian, M. A. Avascular Necrosis of the Femoral Head: Are Any Genes Involved? Arch Bone Jt Surg 3, 149-155 (2015).

19 Wang, Y. et al. Alcohol-induced adipogenesis in bone and marrow: a possible mechanism for osteonecrosis. Clin Orthop Relat Res, 213-224, doi:10.1097/01.blo.0000063602.67412.83 (2003).

20 Kong, X. et al. Ethyl acetate fraction of Huogu formula inhibits adipogenic differentiation of bone marrow stromal cells via the BMP and Wnt signaling pathways. Int J Biol Sci 13, 480-491, doi:10.7150/ijbs.18430 (2017).

$21 \mathrm{Kim}$, Y. H. \& Kim, J. S. Histologic analysis of acetabular and proximal femoral bone in patients with osteonecrosis of the femoral head. J Bone Joint Surg Am 86, 2471-2474, doi:10.2106/00004623200411000-00017 (2004).

22 Calder, J. D., Pearse, M. F. \& Revell, P. A. The extent of osteocyte death in the proximal femur of patients with osteonecrosis of the femoral head. J Bone Joint Surg Br 83, 419-422, doi:10.1302/0301620x.83b3.10793 (2001).

23 Kantor, S. G., Huo, M. H., Huk, O. L. \& Salvati, E. A. Cemented total hip arthroplasty in patients with osteonecrosis. A 6-year minimum follow-up study of second-generation cement techniques. $J$ Arthroplasty 11, 267-271, doi:10.1016/s0883-5403(96)80076-3 (1996).

24 Acurio, M. T. \& Friedman, R. J. Hip arthroplasty in patients with sickle-cell haemoglobinopathy. J Bone Joint Surg Br 74, 367-371, doi:10.1302/0301-620x.74b3.1587879 (1992).

25 Fassihi, S. C. et al. Total Hip Arthroplasty in Patients With Sickle Cell Disease: A Comprehensive Systematic Review. J Arthroplasty 35, 2286-2295, doi:10.1016/j.arth.2020.04.014 (2020).

26 Ilyas, I. \& Al-Mohrej, O. A. High Incidence of Irradiated Cortical Strut Allograft Resorption Following Revision of Femoral Stems. J Arthroplasty 36, 1413-1419, doi:10.1016/j.arth.2020.10.023 (2021).

27 Goldschlager, T., Abdelkader, A., Kerr, J., Boundy, I. \& Jenkin, G. Undecalcified bone preparation for histology, histomorphometry and fluorochrome analysis. J Vis Exp, doi:10.3791/1707 (2010). 
28 Kulak, C. A. \& Dempster, D. W. Bone histomorphometry: a concise review for endocrinologists and clinicians. Arq Bras Endocrinol Metabo/ 54, 87-98, doi:10.1590/s0004-27302010000200002 (2010).

29 Plenk, H., Jr. et al. Magnetic resonance imaging and histology of repair in femoral head osteonecrosis. Clin Orthop Relat Res, 42-53, doi:10.1097/00003086-200105000-00006 (2001).

30 Sissons, H. A., Nuovo, M. A. \& Steiner, G. C. Pathology of osteonecrosis of the femoral head. A review of experience at the Hospital for Joint Diseases, New York. Skeletal Radiol 21, 229-238, doi:10.1007/bf00243063 (1992).

31 Chiba, K., Burghardt, A. J., Osaki, M. \& Majumdar, S. Heterogeneity of bone microstructure in the femoral head in patients with osteoporosis: an ex vivo HR-pQCT study. Bone 56, 139-146, doi:10.1016/j.bone.2013.05.019 (2013).

32 Wang, C. et al. Bone microstructure and regional distribution of osteoblast and osteoclast activity in the osteonecrotic femoral head. PloS one 9, e96361, doi:10.1371/journal.pone.0096361 (2014).

$33 \mathrm{Ma}, \mathrm{J} .-\mathrm{X}$. et al. Bone Microarchitecture and Biomechanics of the Necrotic Femoral Head. Sci Rep 7, 13345-13345, doi:10.1038/s41598-017-13643-2 (2017).

$34 \mathrm{He}, \mathrm{M} .-\mathrm{C}$. et al. Osteoclastic activity was associated with the development of steroid-induced osteonecrosis of femoral head. Artificial Cells, Nanomedicine, and Biotechnology 48, 1036-1046, doi:10.1080/21691401.2020.1774596 (2020).

35 Fu, D., Yang, S., Lu, J., Lian, H. \& Qin, K. LncRNA NORAD promotes bone marrow stem cell differentiation and proliferation by targeting miR-26a-5p in steroid-induced osteonecrosis of the femoral head. Stem Cell Res Ther 12, 18, doi:10.1186/s13287-020-02075-x (2021).

36 Yoon, B. H. et al. Etiologic Classification Criteria of ARCO on Femoral Head Osteonecrosis Part 1: Glucocorticoid-Associated Osteonecrosis. J Arthroplasty 34, 163-168.e161, doi:10.1016/j.arth.2018.09.005 (2019).

37 Fukushima, W. et al. The effect of alcohol intake and the use of oral corticosteroids on the risk of idiopathic osteonecrosis of the femoral head: a case-control study in Japan. Bone Joint J 95-b, 320-325, doi:10.1302/0301-620x.95b3.30856 (2013).

38 Yoon, B. H. et al. Etiologic Classification Criteria of ARCO on Femoral Head Osteonecrosis Part 2: Alcohol-Associated Osteonecrosis. J Arthroplasty 34, 169-174.e161, doi:10.1016/j.arth.2018.09.006 (2019).

39 Zhao, D. et al. Guidelines for clinical diagnosis and treatment of osteonecrosis of the femoral head in adults (2019 version). J Orthop Translat 21, 100-110, doi:10.1016/j.jot.2019.12.004 (2020). 
40 Sugano, N. et al. The 2001 revised criteria for diagnosis, classification, and staging of idiopathic osteonecrosis of the femoral head. J Orthop Sci 7, 601-605, doi:10.1007/s007760200108 (2002).

41 Tjong, W., Kazakia, G. J., Burghardt, A. J. \& Majumdar, S. The effect of voxel size on high-resolution peripheral computed tomography measurements of trabecular and cortical bone microstructure. Med Phys 39, 1893-1903, doi:10.1118/1.3689813 (2012).

42 Tassani, S. et al. Dependence of trabecular structure on bone quantity: a comparison between osteoarthritic and non-pathological bone. Clin Biomech (Bristol, Avon) 26, 632-639, doi:10.1016/j.clinbiomech.2011.01.010 (2011).

43 Bouxsein, M. L. et al. Guidelines for assessment of bone microstructure in rodents using microcomputed tomography. J Bone Miner Res 25, 1468-1486, doi:10.1002/jbmr.141 (2010).

$44 \mathrm{Li}, \mathrm{G}$. et al. Identical subchondral bone microarchitecture pattern with increased bone resorption in rheumatoid arthritis as compared to osteoarthritis. Osteoarthritis Cartilage 22, 2083-2092, doi:10.1016/j.joca.2014.08.015 (2014).

45 Altman, D. G., Gore, S. M., Gardner, M. J. \& Pocock, S. J. Statistical guidelines for contributors to medical journals. Br Med J (Clin Res Ed) 286, 1489-1493, doi:10.1136/bmj.286.6376.1489 (1983).

46 Yoon, B. H. et al. The 2019 Revised Version of Association Research Circulation Osseous Staging System of Osteonecrosis of the Femoral Head. J Arthroplasty 35, 933-940, doi:10.1016/j.arth.2019.11.029 (2020).

47 Weinstein, R. S. True strength. J Bone Miner Res 15, 621-625, doi:10.1359/jbmr.2000.15.4.621 (2000).

48 Dalle Carbonare, L. \& Giannini, S. Bone microarchitecture as an important determinant of bone strength. J Endocrinol Invest 27, 99-105, doi:10.1007/bf03350919 (2004).

49 Fazzalari, N. L. \& Parkinson, I. H. Femoral trabecular bone of osteoarthritic and normal subjects in an age and sex matched group. Osteoarthritis Cartilage 6, 377-382, doi:10.1053/joca.1998.0141 (1998).

50 van der Harst, M. R. et al. An integral biochemical analysis of the main constituents of articular cartilage, subchondral and trabecular bone. Osteoarthritis Cartilage 12, 752-761, doi:10.1016/j.joca.2004.05.004 (2004).

51 Ma, J. X. et al. Bone Microarchitecture and Biomechanics of the Necrotic Femoral Head. Sci Rep 7, 13345, doi:10.1038/s41598-017-13643-2 (2017).

52 Baba, S. et al. Quantitative evaluation of bone-resorptive lesion volume in osteonecrosis of the femoral head using micro-computed tomography. Joint Bone Spine 87, 75-80, doi:10.1016/j.jbspin.2019.09.004 (2020). 
53 Seeman, E. \& Delmas, P. D. Bone quality-the material and structural basis of bone strength and fragility. N Engl J Med 354, 2250-2261, doi:10.1056/NEJMra053077 (2006).

54 Issever, A. S. et al. A micro-computed tomography study of the trabecular bone structure in the femoral head. J Musculoskelet Neuronal Interact 3, 176-184 (2003).

55 Lespasio, M. J., Sodhi, N. \& Mont, M. A. Osteonecrosis of the Hip: A Primer. Perm J 23, doi:10.7812/tpp/18-100 (2019).

56 Kahler-Quesada, A. M. et al. Voluntary Chronic Heavy Alcohol Consumption in Male Rhesus Macaques Suppresses Cancellous Bone Formation and Increases Bone Marrow Adiposity. Alcohol Clin Exp Res 43, 2494-2503, doi:10.1111/acer.14202 (2019).

57 Johnston, J. C., Haile, A., Wang, D., Ronnett, G. \& Jones, L. C. Dexamethasone treatment alters function of adipocytes from a mesenchymal stromal cell line. Biochem Biophys Res Commun 451, 473479, doi:10.1016/j.bbrc.2014.07.063 (2014).

58 Sheng, $\mathrm{H}$. et al. Pathomorphological changes of bone marrow adipocytes in process of steroidassociated osteonecrosis. Int J Clin Exp Pathol 6, 1046-1050 (2013).

59 Weinstein, R. S. Clinical practice. Glucocorticoid-induced bone disease. N Eng/ J Med 365, 62-70, doi:10.1056/NEJMcp1012926 (2011).

60 Luo, Z. et al. Cellular and molecular mechanisms of alcohol-induced osteopenia. Cell Mol Life Sci 74, 4443-4453, doi:10.1007/s00018-017-2585-y (2017).

61 Dai, J. et al. Chronic alcohol ingestion induces osteoclastogenesis and bone loss through IL-6 in mice. J Clin Invest 106, 887-895, doi:10.1172/JCI10483 (2000).

62 Chernetsky, S. G. et al. Pathologic features in steroid and nonsteroid associated osteonecrosis. Clin Orthop Relat Res, 149-161 (1999).

63 Wei, Q. et al. [Microarchitecture features and pathology of necrotic region in patients with steroidinduced and alcohol-induced osteonecrosis of femoral head]. Zhongguo Xiu Fu Chong Jian Wai Ke Za Zhi 32, 866-872, doi:10.7507/1002-1892.201711130 (2018).

64 Mont, M. A., Jones, L. C., Einhorn, T. A., Hungerford, D. S. \& Reddi, A. H. Osteonecrosis of the femoral head. Potential treatment with growth and differentiation factors. Clin Orthop Relat Res, S314-335 (1998).

65 Liu, B. \& Goodwin, J. E. The Effect of Glucocorticoids on Angiogenesis in the Treatment of Solid Tumors. J Cell Signal 1, $42-49$ (2020).

66 Guo, Y. J. et al. Muscone exerts protective roles on alcohol-induced osteonecrosis of the femoral head. Biomed Pharmacother 97, 825-832, doi:10.1016/j.biopha.2017.11.025 (2018). 
67 Zhang, Y., Yuan, H., Sun, Y., Wang, Y. \& Wang, A. The effects of ethanol on angiogenesis after myocardial infarction, and preservation of angiogenesis with rosuvastatin after heavy drinking. Alcohol 54, 27-32, doi:10.1016/j.alcohol.2016.05.003 (2016).

68 Baum, T. et al. Automated 3D trabecular bone structure analysis of the proximal femur-prediction of biomechanical strength by CT and DXA. Osteoporos Int 21, 1553-1564, doi:10.1007/s00198-009-1090-z (2010).

69 Krug, R. et al. Feasibility of in vivo structural analysis of high-resolution magnetic resonance images of the proximal femur. Osteoporos Int 16, 1307-1314, doi:10.1007/s00198-005-1907-3 (2005).

70 Mulder, L., van Rietbergen, B., Noordhoek, N. J. \& Ito, K. Determination of vertebral and femoral trabecular morphology and stiffness using a flat-panel C-arm-based CT approach. Bone 50, 200-208, doi:10.1016/j.bone.2011.10.020 (2012).

71 Chiba, K., Ito, M., Osaki, M., Uetani, M. \& Shindo, H. In vivo structural analysis of subchondral trabecular bone in osteoarthritis of the hip using multi-detector row CT. Osteoarthritis Cartilage 19, 180185, doi:10.1016/j.joca.2010.11.002 (2011).

\section{Figures}



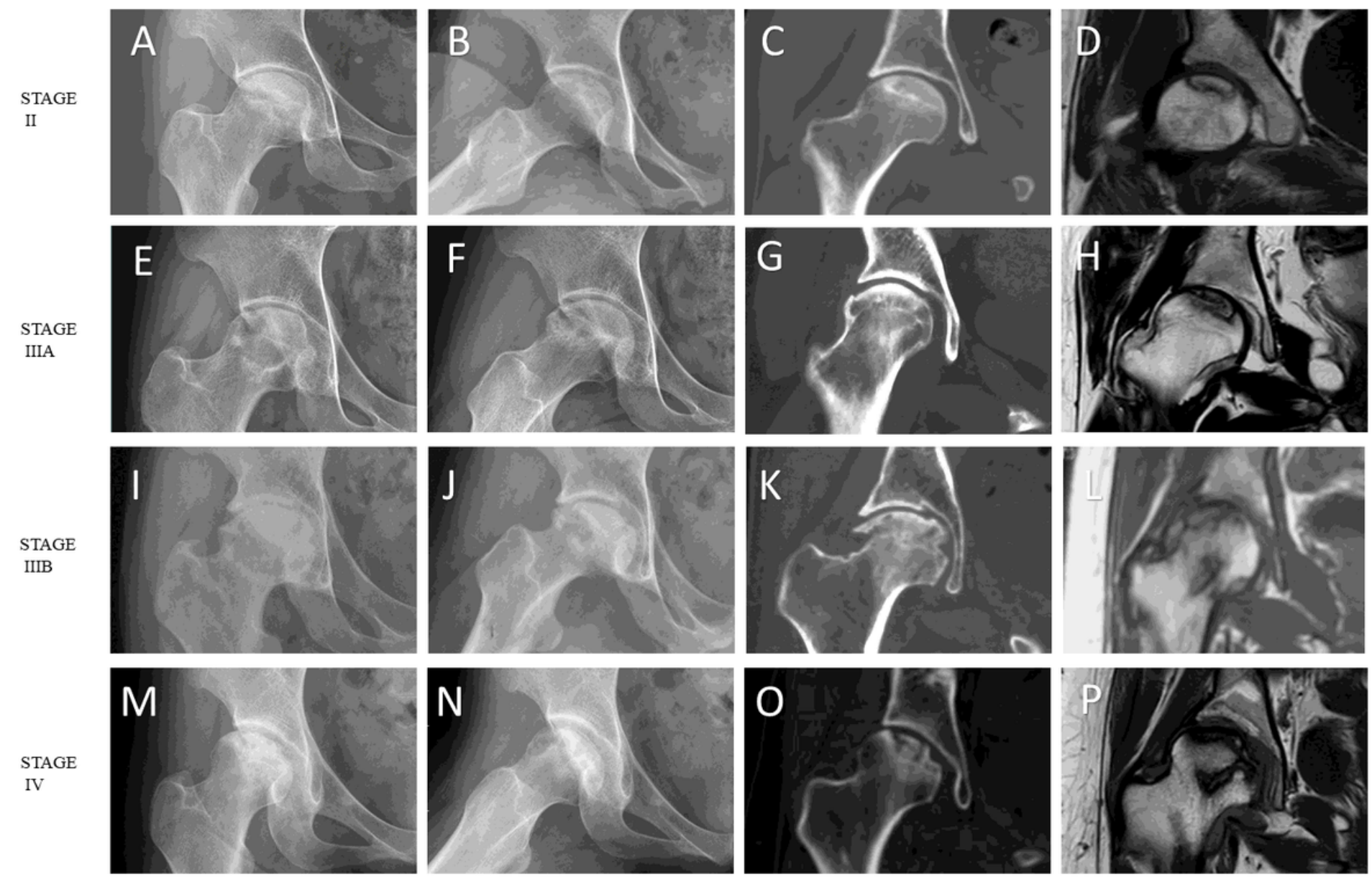

Figure 1

The cases in this study were all ARCO stage II, IIIA, IIIB or IV, with severe pain or severe hip dysfunction
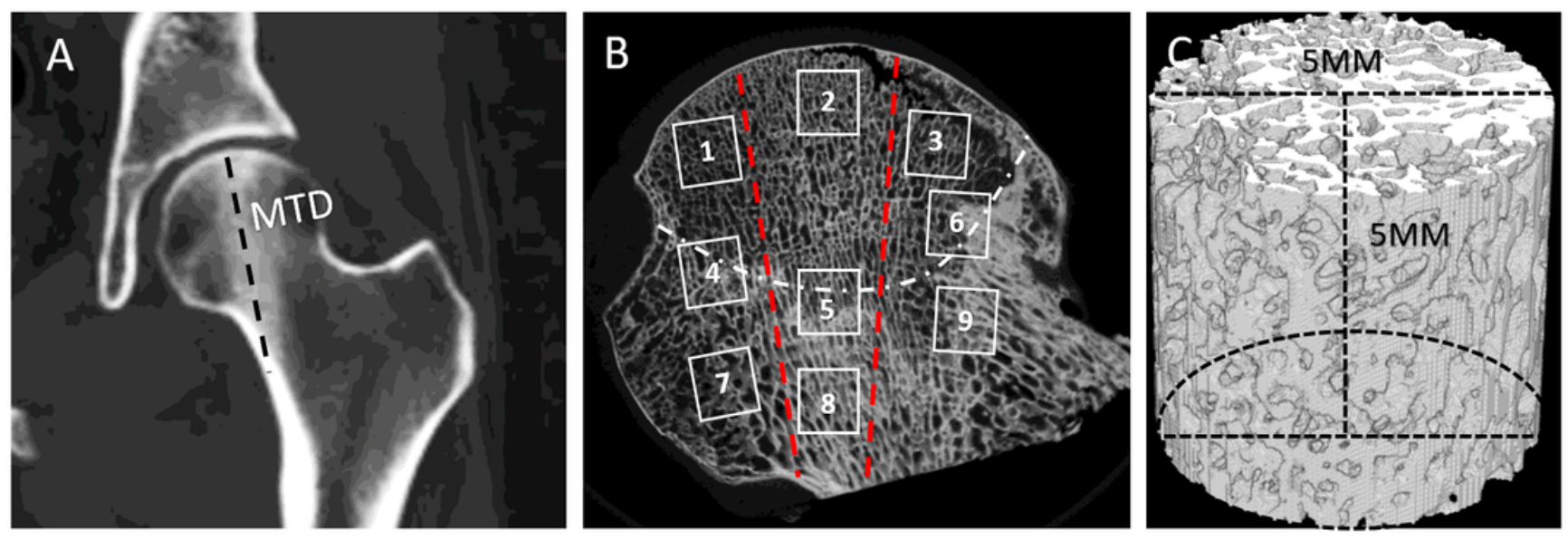

Figure 2 
Orientations of all femoral heads were adjusted based on anatomical landmarks using the fovea capitis femoris and the main trabeculae direction (MTD), where the principal compressive trabeculae were located
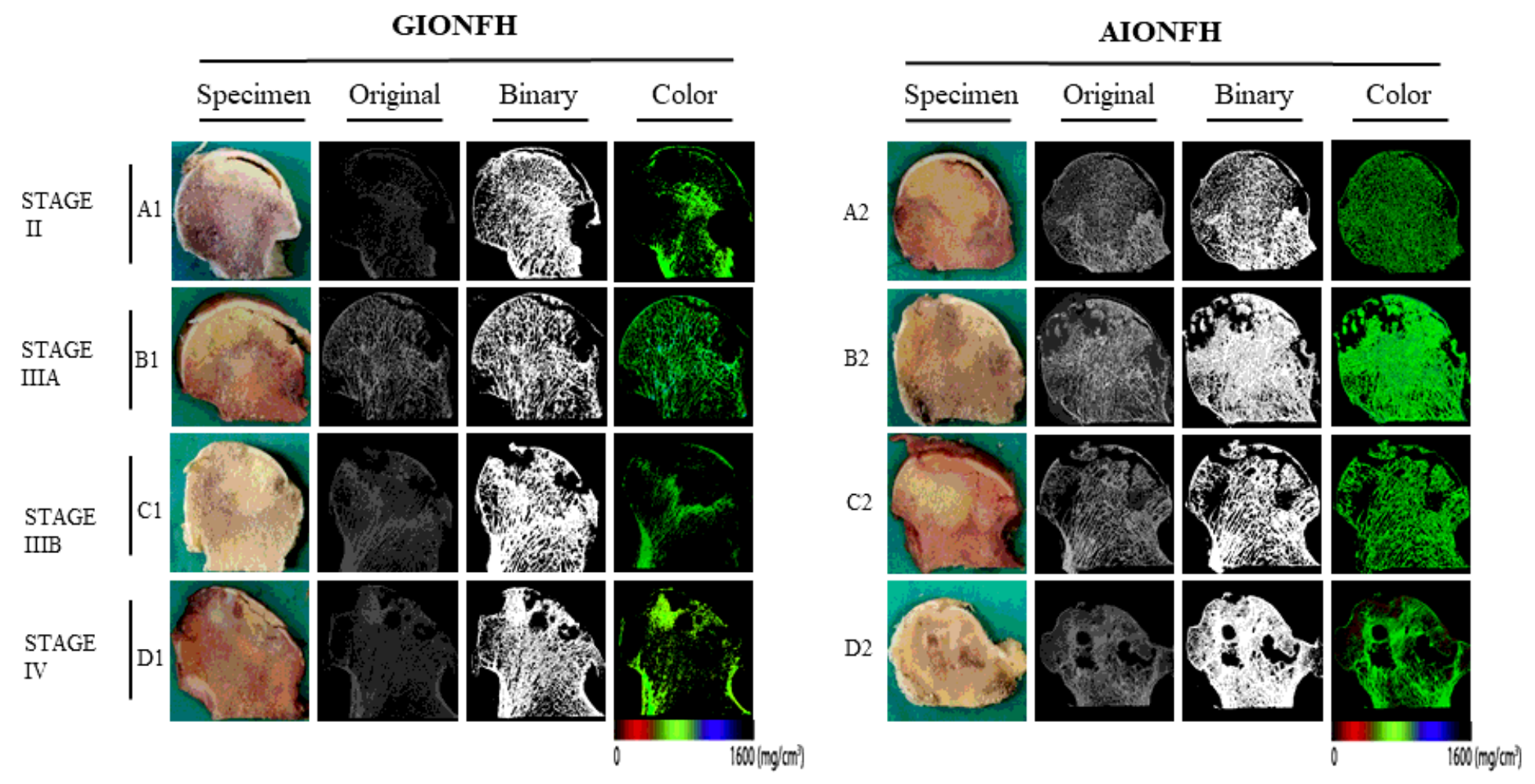

Figure 3

After micro-CT scanning, all femoral heads were cut in half with a diamond saw, coronally separating the anterior hemisphere from the posterior hemisphere 


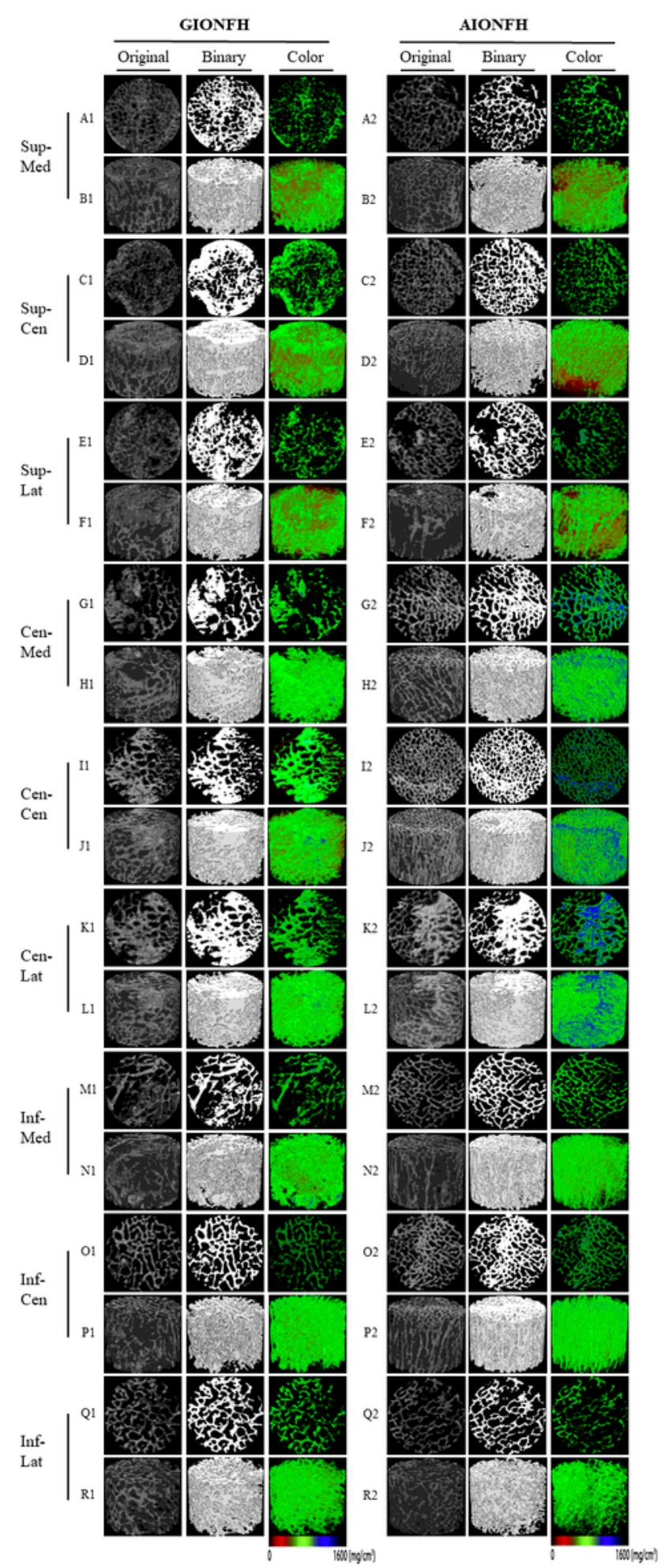

Figure 4

There were significantly lower values of DA in GIONFH, compared to AIONFH. Similarly, in Sup-Cen and Sup-Lat regions, none of the microarchitecture parameters differed significantly between GIONFH and AIONFH, with the exception of SMI 
A

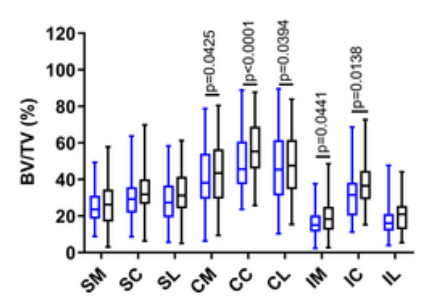

$E$

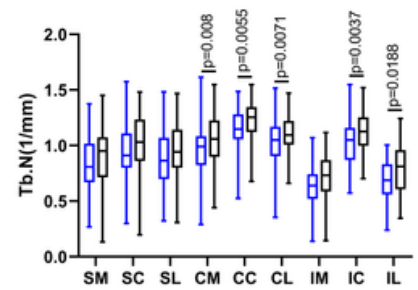

B

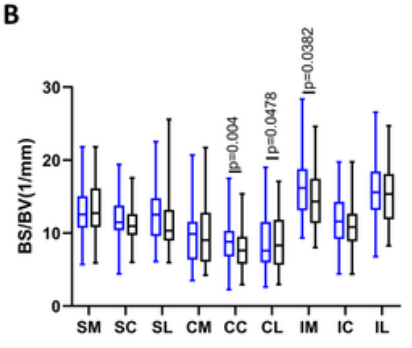

$F$

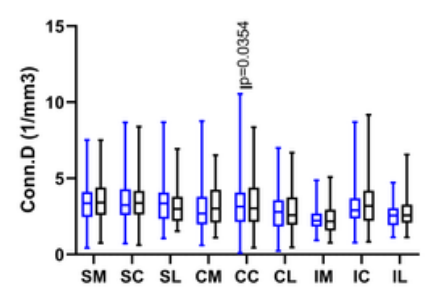

C

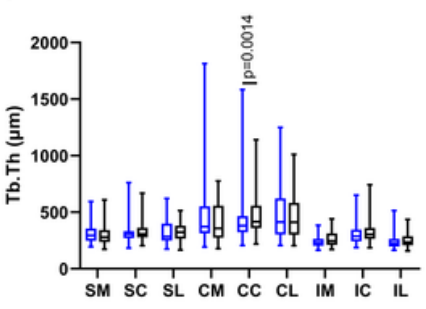

G

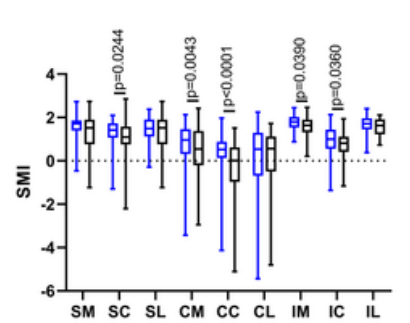

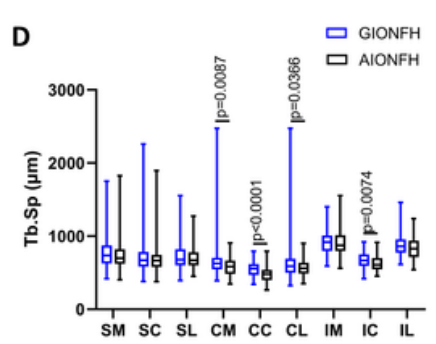

H

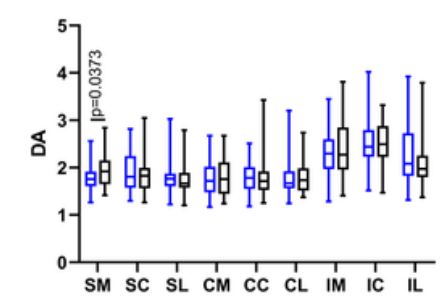

\section{Figure 5}

There were significantly lower values of BV/TV and Tb.N, higher values of Tb.Sp and SMI in GIONFH, compared to AIONFH. In Inf-Lat region, none of the microarchitecture parameters differed significantly between GIONFH and AIONFH, except for Tb.N
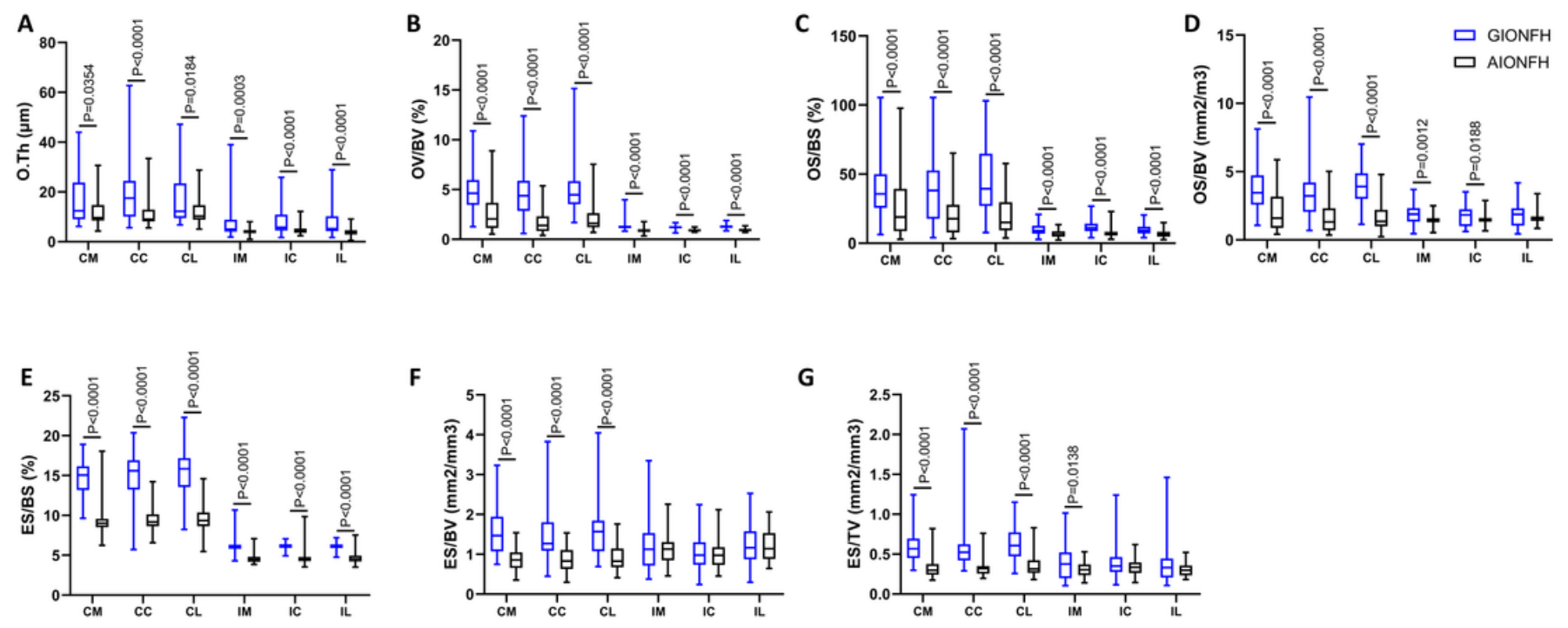

\section{Figure 6}

In the necrotic-normal junction region, including Cen-Med, Cen-Cen and Cen-Lat regions, all the bone remodeling parameters were significantly higher in GIONFH, compared with AIONFH 
GIONFH
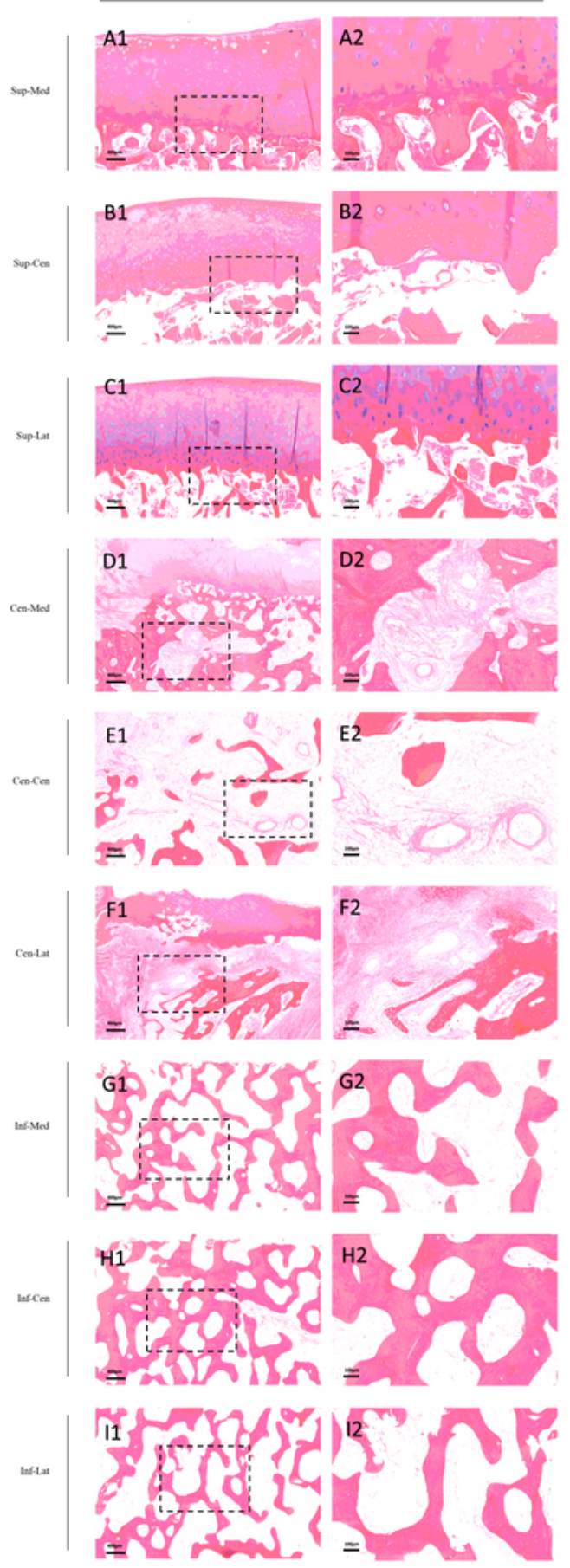

AIONFH
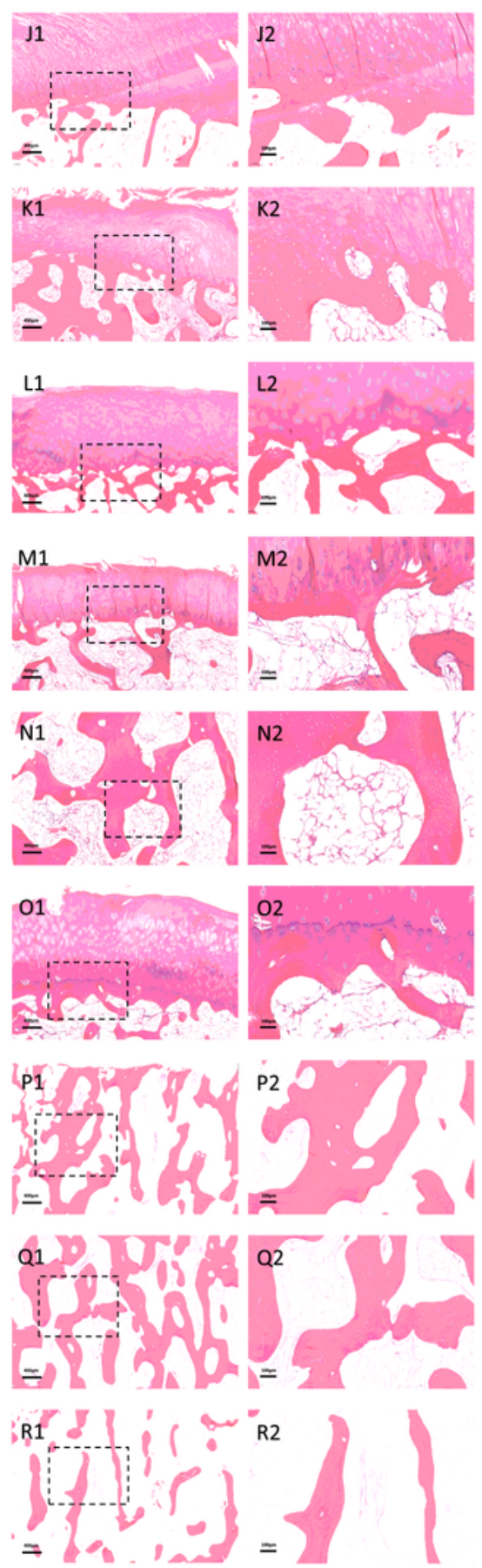

\section{Figure 7}

Hematopoietic cells disappeared and adipose nucleus staining was absent in the bone marrow, indicating the necrosis of bone marrow 

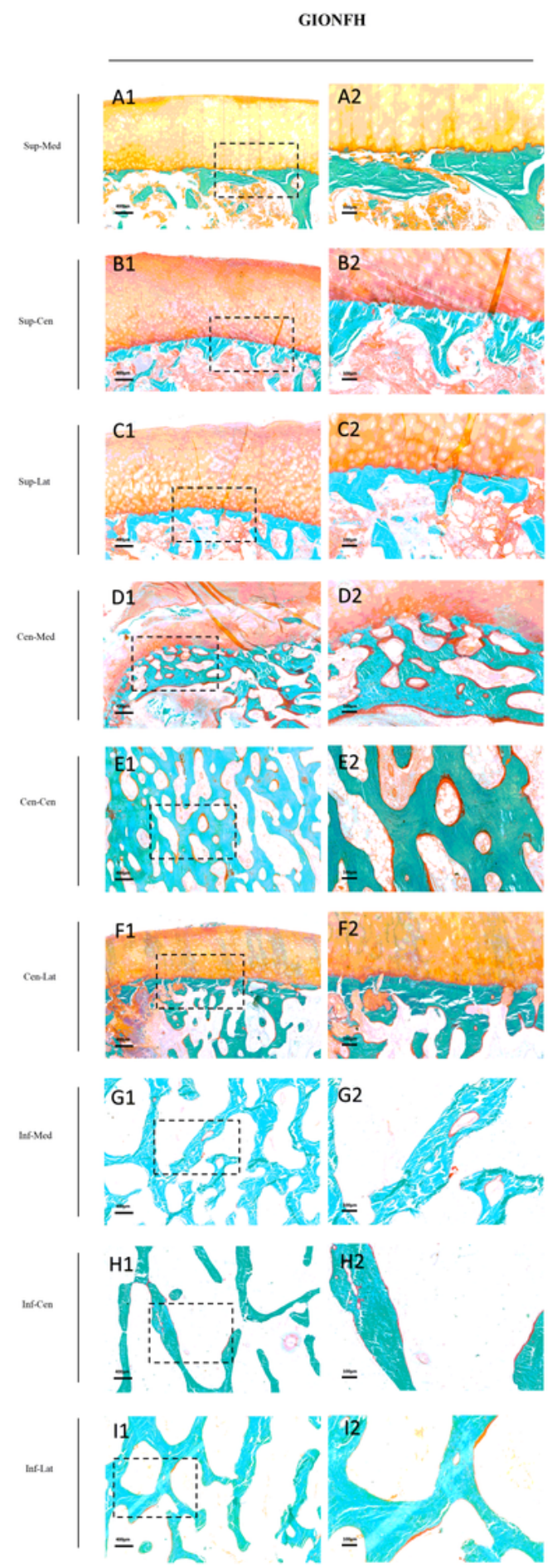

AIONFH
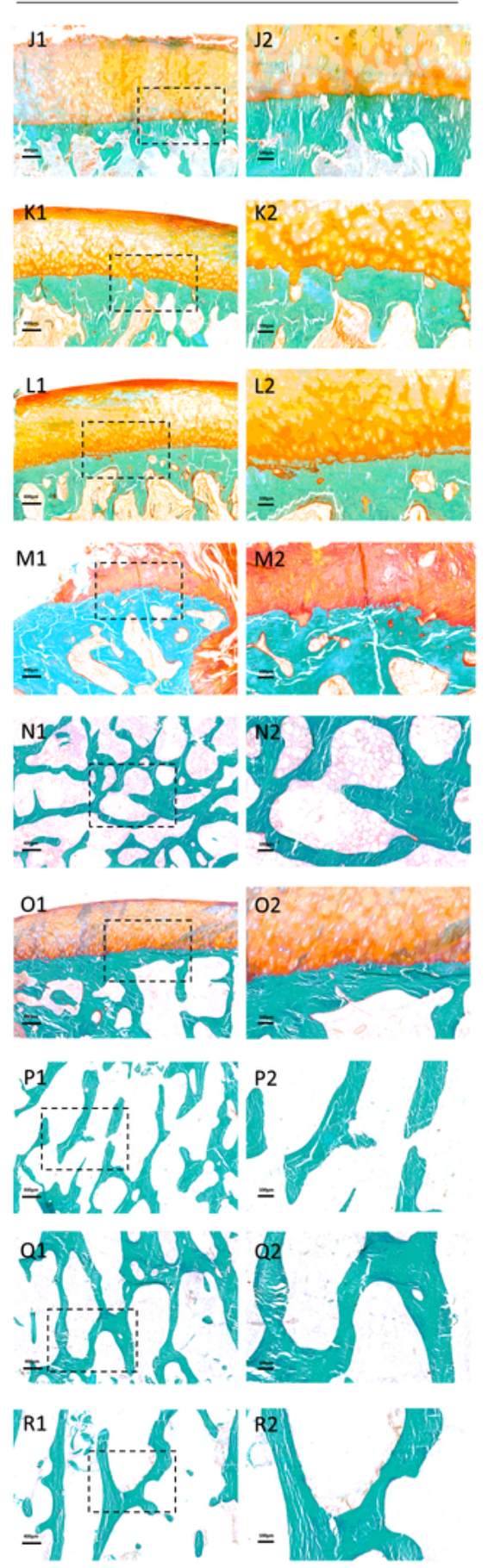

\section{Figure 8}

The bone resorption parameter ES/BS was significantly elevated in GIONFH. No significant differences were observed in ES/BV and ES/TV. In Inf-Lat region, there were higher values of O.Th, OV/BV, OS/BS in GIONFH, indicating a more active bone formation status 